\title{
BEAM STEERING IN SMART ANTENNAS BY USING LOW COMPLEX
} ADAPTIVE ALGORITHMS

\author{
Amarnadh Poluri ${ }^{1}$, Ashish kumar ${ }^{2}$ \\ ${ }^{1}$ Student, ${ }^{2}$ Asst Professor, Department of Electronics and Communications Engineering, Vignan's Institute Of Information \\ Technology, Duvvada, Visakhapatnam, Andhra Pradesh, India. \\ amarnadh401@gmail.com,.ashishkumar.ambastha@gmail.com
}

\begin{abstract}
Array antenna systems are often used to enhance the received signal to interference and noise ratio when the signal operates in heavily jammed environment. Proper modeling of the received data at different antenna elements is important when evaluating the performance of this system, especially when both the signal and interference have wide frequency bands.. The antenna output is the linear combination of data from all the antenna elements. In conventional narrowband beam forming, time sequences at different antenna elements are related by some fixed phase shift. The phase shift is determined by the wave forms direction of arrival (DOA).

In this paper, an efficient method for the pattern synthesis of the linear antenna arrays with the prescribed nulling and steering lobe is presented. The proposed method is based on Least Mean Square (LMS) algorithm provide a comprehensive and detailed treatment of the signal model used for beam forming, as well as, describing adaptive algorithms to adjust the weights of an array. In order to improve the convergence rate of LMS algorithm in smart antenna system, in this paper we proposes a new normalized LMS (NLMS) algorithm, This new algorithm can be treated as a block based simplification of NLMS algorithm which gives satisfactory performance in certain applications in comparison with conventional NLMS recursion, i.e., BBNLMS algorithm. By taking advantage of spatial filtering, the proposed scheme promises to reduce the bandwidth required for transmitting data by improving convergence rate. The performance of the BBNLMS algorithm in the presence of Multi-path effects and multiple users is analyzed using MATLAB simulations. The simulations when compared to that of the LMS algorithm, the results suggest that BBNLMS algorithm can improve the convergence rate and lead to better system efficiency.
\end{abstract}

Keywords: BBNLMS, Convergence Rate, DOA, LMS, NLMS, Smart antenna. $* * *$

\section{INTRODUCTION}

The performance of a communication system in an interference environment can be severely degraded if the received signal to interference ratio is unacceptably low. Smart antenna is the critical technique of the third mobile communication, while the core of smart antenna is adaptive algorithm research. Smart antennas can be used to achieve different benefits. Among those the most important is higher network capacity [1], [2] by precise control of signal null equality and mitigation of interference combine to frequency reuse reduction distance improving capacity. The term adaptive antenna is used for a phased array when the weight of each element is applied in a dynamic fashion. The amount of weighting on each channel is not fixed at the time of the array design, but rather decided by the system at the time of processing the signals to meet required objectives.

In other words, the array pattern adapts to the situation and the adaptive process is under control of the system. For example, consider the situation of a communication system operating in the presence of a directional interference operating at the carrier frequency used by the desired signal, and the performance measure is to maximize the output signal to noise ratio (SNR), in such systems the desirable is output SNR should be maximized by cancelling the directional interference using optimal antennas. The antenna pattern in this case has a main beam pointed in the desired signal direction, and has a null in the direction of the interference. Assume that the interference is not stationary but moving slowly. If optimal performance is to be maintained, the antenna pattern needs to adjust so that the null position remains in the moving interference direction.

In this paper we propose a simple and novel adaptive algorithm for steering the antenna beam electronically. Generally LMS algorithm is widely used in adaptive filter due to its relatively low computational complexity, good stability properties, and relatively good robustness against implementation errors. However, the least mean square (LMS) algorithm has poor convergence rate, which reduces the system performance. In order increase the convergence rate, LMS algorithm is modified by normalization, which is known as normalized LMS (NLMS)[8]. In our work NLMS algorithm 
to increase the convergence rate and reducing computational complexity Block processing of data in adaptive filters significantly reduces computational burden and improves convergence performance [9]. In literature several smart antenna processing techniques were presented [10]-[17], to the best of author's knowledge reduction of computational complexity in the array processing is not addressed. Finally in our simulations we considered two received signals and three directions of arrivals (DOAs). Simulation results confirm that the proposed BBNLMS based beam steering is superior than conventional LMS algorithm in terms of complexity and convergence rate. Normalization of step size in LMS algorithm improves the convergence rate and decreases excess mean square error, by exploiting this we have implemented beam forming using NLMS algorithms, i.e., NLMS algorithm and BBNLMS algorithm.

\section{ADAPTIVE BEAM FORMING}

In certain applications the gain of a single antenna may not sufficient, array antennas plays a vital role in such situations. In array antennas the beam can be steered in two ways, ie., mechanical steering and electronic steering. Adaptive beam forming can be performed in many ways using adaptive algorithms. Several adaptive algorithms are presented in literature. Most of the algorithms are concerned with the maximization of the SNR. A functional diagram of an adaptive array system is shown in fig 1

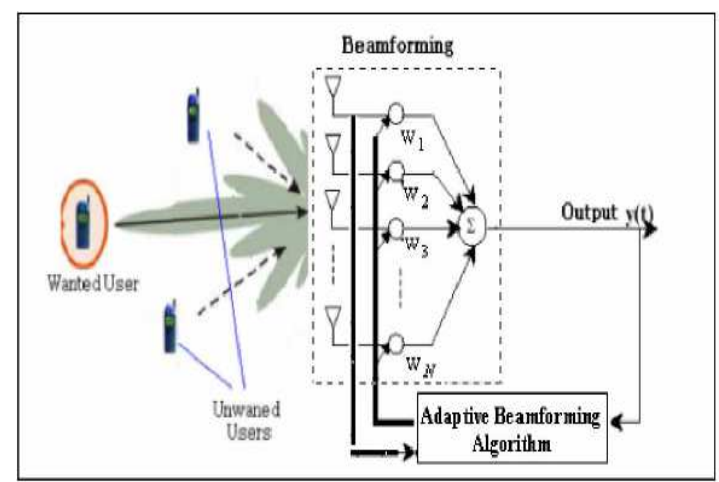

Fig-1: Functional block diagram of an adaptive array system

Adaptive array systems can locate and track signals (users and interferers) and dynamically adjust the antenna pattern to enhance reception while minimizing interference using signal processing algorithms.

After the system down converts the received signals to baseband and digitizes them, it locates the signal of interest (SOI) using the DOA algorithm, and it continuously tracks the SOI and signal not of interest (SNOI)s by dynamically changing the complex weights (amplitudes and phases of the antenna elements). Basically, the DOA computes the direction-of-arrival of all the signals by computing the time delays between the antenna elements, and afterward, the adaptive algorithm, using a cost function, computes the appropriate weights that result in an optimum radiation pattern. Because adaptive arrays are generally more digital processing intensive and require a complete RF portion of the transceiver behind each antenna element, they tend to be more expensive than switched-beam systems. Adaptive arrays utilize sophisticated signal-processing algorithms to continuously distinguish between desired signals, multipath, and interfering signals, as well as calculate their DOA. This approach updates its transmit strategy continuously based on changes in both the desired and interfering signal locations.

In adaptive beam forming techniques, two main strategies are distinguished. The first one is based on the assumption that part of the desired signal is already known through the use of a training sequence. This known signal is then compared with what is received, and the weights are then adjusted to minimize the Mean Square Error (MSE) between the known and the received signals. In this way, the beam pattern can be adjusted to null the interferers. This approach optimizes the signal-to-interference ratio (SIR), and is applicable to nonline-of-sight (NLOS) environments. Since the weights are updated according to the incoming signals, not only the interference is reduced but the multipath fading is also mitigated. In the second one, the directions of arrivals from all sources transmitting signals to the array antenna are first identified. The complex weights are then adjusted to produce a maximum toward the desired angle and null toward interfering signals. This strategy may turn out to be deficient in practical scenarios where there are too many DOAs due to multi paths, and the algorithms are more likely to fail in properly detecting them. This is more likely to occur in NLOS environments where there are many local scatters close to the users and the base station, thus resulting in a wider spread of the angle of arrival. Another significant advantage of the adaptive antenna systems is the ability to share spectrum. Because of the accurate tracking and robust interference rejection capabilities, multiple users can share the same conventional channel within the same cell. System capacity increases through lower intercell frequency reuse patterns as well as intra-cell frequency reuse.

\section{ADAPTIVE ALGORITHMS}

An adaptive filter is a transversal filter trained by an adaptive algorithm. The algorithm updates the weights at each iteration by estimating the gradient of the quadratic MSE surface and then moving the weights in the negative direction of the gradient by a minute amount. The constant that determines this amount is referred to as the step size $(\mu)$. When this step size is small enough, the process leads these estimated weights to the optimal weights. The convergence and transient behavior of these weights along with their covariance characterize the LMS algorithm and the way the step size and 
process of gradient estimation affect these parameters are of great practical importance.

\subsection{The Least Mean Square (LMS) Algorithm:}

The LMS algorithm is probably the most widely used adaptive filtering algorithm, being employed in several communication systems. It has gained popularity due to its low computational complexity and proven robustness. It incorporates new observations and iteratively minimizes linearly the meansquare error. This algorithm is based on the knowledge of arriving signal. The knowledge of the received signal eliminates the need for beam forming, but the reference can also be a vector which is somewhat correlated with the received signal. As shown in Figure 1, an adaptive beam former consists of multiple antennas, complex weights, the function of which is to amplify or attenuate and delay the signals from each antenna element, and a summer to add all of the processed signals, in order to tune out the signals not of interest, while enhancing the signal of interest. Hence, beam forming is some time referred to as spatial directions are filtered out, while others are amplified. The response of the LMS is as follows,

$$
\mathrm{w}^{\prime}(\mathrm{n}+1)=\mathrm{w}^{\prime}(\mathrm{n})+\mu \mathrm{u}(\mathrm{n}) \mathrm{e}^{*}(\mathrm{n})
$$

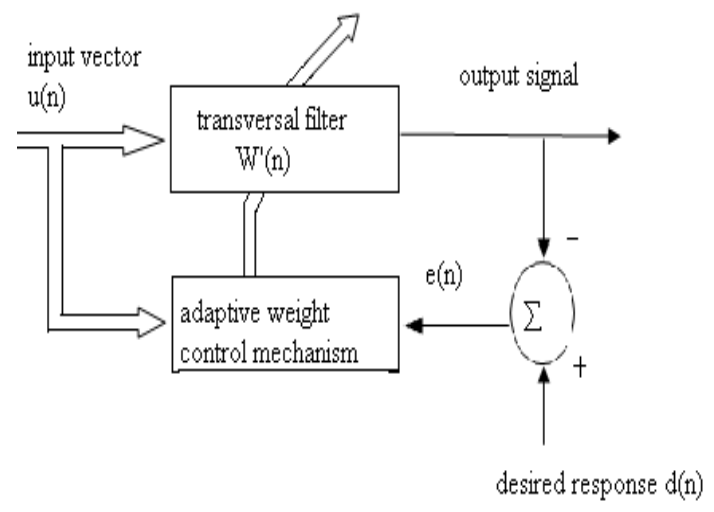

Fig-2: Block diagram of adaptive transversal filter

\subsection{Normalized Least Mean Square (NLMS)}

\section{Algorithm:}

In structural terms, the normalized LMS filter is exactly the same as the standard LMS filter. In the standard form of a least mean square (LMS) filter studied in earlier the adjustment applied to the tap-weight vector of the filter at iteration $n+1$ consists of the product of three terms.

- The step-size parameter $\mu$, which is under the designer's control.

- The tap-input vector $\mathrm{u}(\mathrm{n})$, which is supplied by a source of information.
- The estimation error e(n) for real-valued data, or its complex conjugate $e *(n)$ for complex-valued data, which is calculated at iteration $n$.

The adjustment is directly proportional to the tap-input vector $\mathrm{u}(\mathrm{n})$. Therefore, when $\mathrm{u}(\mathrm{n})$ is large, the LMS filter suffers from a gradient noise amplification problem. To overcome this difficulty, we may use the normalized LMS filter. In particular, the adjustment applied to the tap-weight vector at iteration $n+1$ is "normalized" with respect to the squared Euclidean norm of the tap-input vector $u(n)$ at iteration $n$ hence the term "normalized". The normalized LMS filter is manifestation of the principle of minimal disturbance, which may be stated as follows. From one iteration to the next, the weight vector of an adaptive filter should be changed in a minimal manner, subject to a constraint imposed on the updated filter's output. The response of the NLMS algorithm is as follows,

$$
\begin{gathered}
\hat{w}_{(n+1)} \hat{w}_{(n)+\frac{\not p}{p+\left.n u[n]\right|^{2}} u(n) * e(n)} \\
\text { Here } e(n)=d(n)-\hat{w}_{H(n)} u(n)
\end{gathered}
$$

\subsection{Block based normalized LMS algorithm (BBLMS):}

In the conventional and normalized LMS algorithms described earlier, the tap weights (free parameters) of a finite-duration impulse response (FIR) filter are adapted in the time domain. Recognizing that the Fourier transforms maps time-domain signals into the frequency domain and that the inverse Fourier transform provides the inverse mapping that takes us back into the time domain, we see that it is equally feasible to perform the adaption of filter parameters in the frequency domain. In such a case, we speak of frequency -domain adaptive filtering (FDAF), There are two main reasons for seeking adaption in the frequency domain in one form or another, Selforthogonalizing adaptive filtering, mechanized in a different way from that is used to improve the convergence performance of the conventional LMS algorithm. And also a more uniform convergence rate is attained by exploiting the orthogonality properties of the discrete Fourier transform (DFT) and related discrete transforms. 


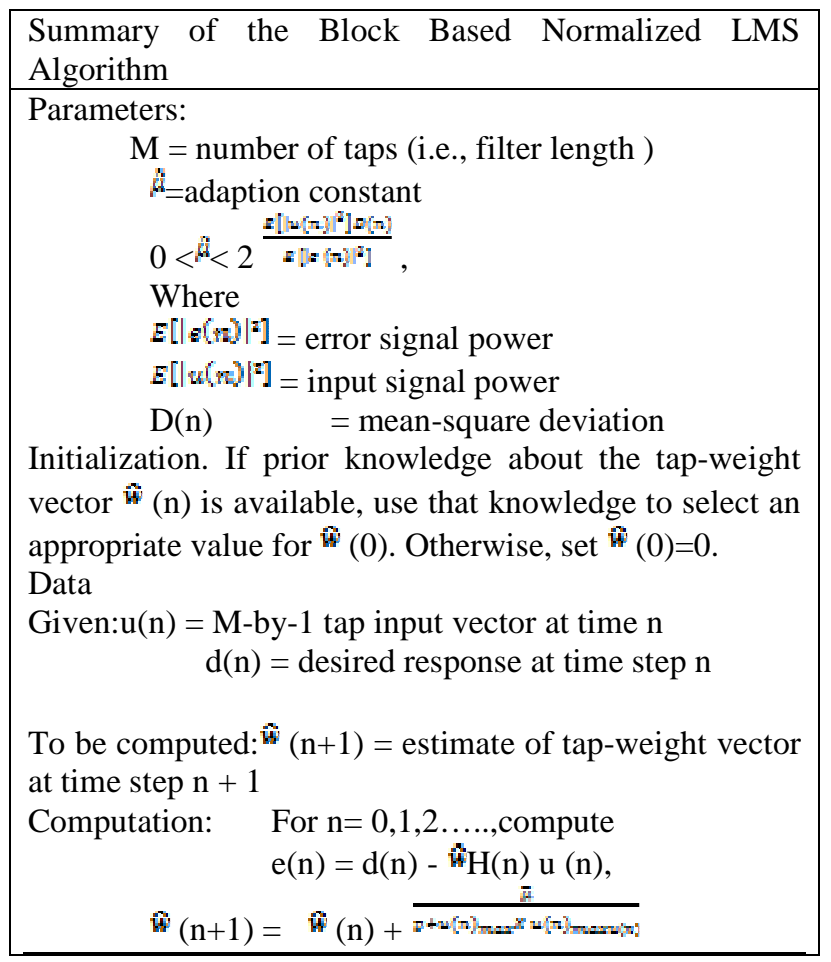

Fig-3: Block Based Normalized LMS Algorithm

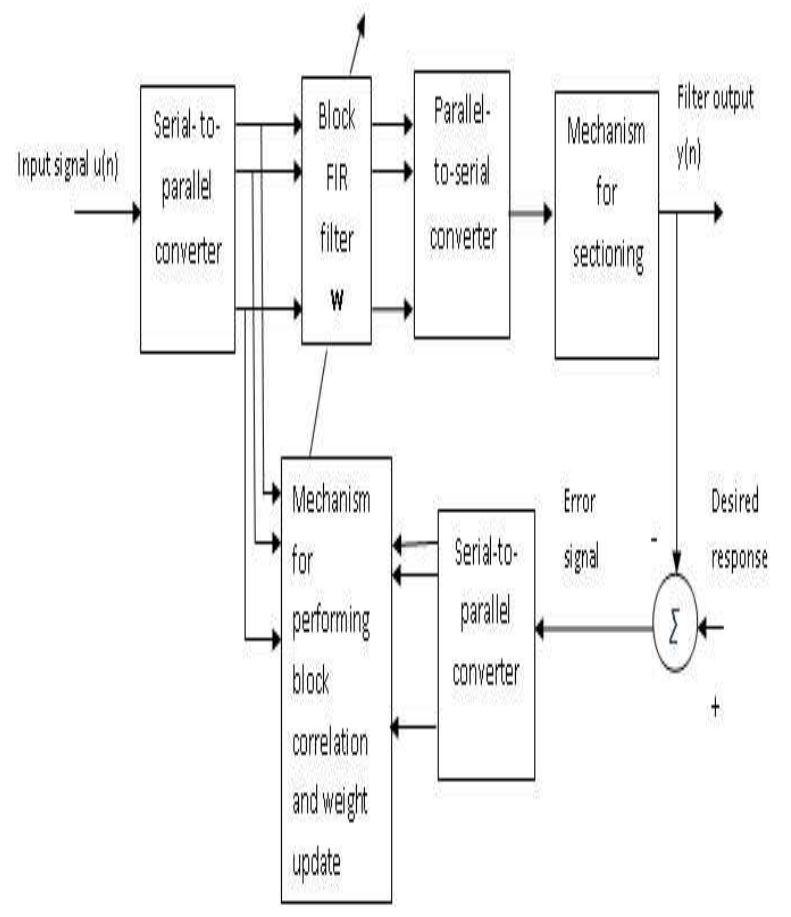

Fig-4: Block-based adaptive filter

In a block adaptive filter, the incoming data sequence $u(n)$ is sectioned into L-point blocks by means of a serial-to -parallel converter, and the blocks of input data so produced are applied to an FIR filter of length $\mathrm{M}$, one block at a time. The tap weights of the filter proceeds on a block-by-block basis rather than on a sample-by-sample basis as in the conventional LMS algorithm.

\section{SIMULATION RESULTS}

The performance of the LMS, NLMS, BB-NLMS algorithms are examined in terms of convergence rate, number of active taps used and beam pattern. In order to replicate realistic mobile environments, for those simulations with more than one multipath, each multipath experience a different gain, which contains both amplitude and phase components. The results will be accompanied by discussions of the observations made and in addition comparisons will be made to that of results achieved. The mean square error for various algorithms is shown in Fig. From the figure is clear that the error amplitude decreases as we are moving from LMS to BBNLMS algorithm. By keen observation of Fig. we can say that convergence starts for LMS approximately after 70th sample, where as for NLMS algorithm it is approximately after 30th sample, superiorly for BBNLMS convergence starts approximately after 15th sample. More ever among all algorithms BBNLMS has minimum error level. The main goal of this project is to develop and study the effects of the proposed algorithms for Smart Antenna systems. To critically examine the performance of the proposed algorithm, the following test cases were designed:

- One White signal with one DOA

- One White Signal with 3 DOA

- Two White Signals with 1 DOA each

- Two White Signals with Three DOAs each different algorithm.

The selection of test cases enables us to investigate multipath and multi-user effects in mobile communication system. The first simulation investigated was the reception of one signal with one path, which arrives at the base station at angle of 600. A gain with amplitude of 0.5 was introduced to the input signal as it was propagated to the antenna. The simulation was conducted for the following threshold control, $\alpha$, values of 0.1 , 0.5 and 1.0.This section we consider three cases. These are Beam forming using LMS algorithm: one white signal with one DOA The convergence characteristics of the three beam forming algorithms are shown in figure 5.1. From the figure it is clear that NLMS convergence faster than LMS. Whereas BBNLMS convergence faster than NLMS. Normalized algorithms convergences faster than conventional LMS algorithm because of the normalization term in the experiences large value in the denominator 


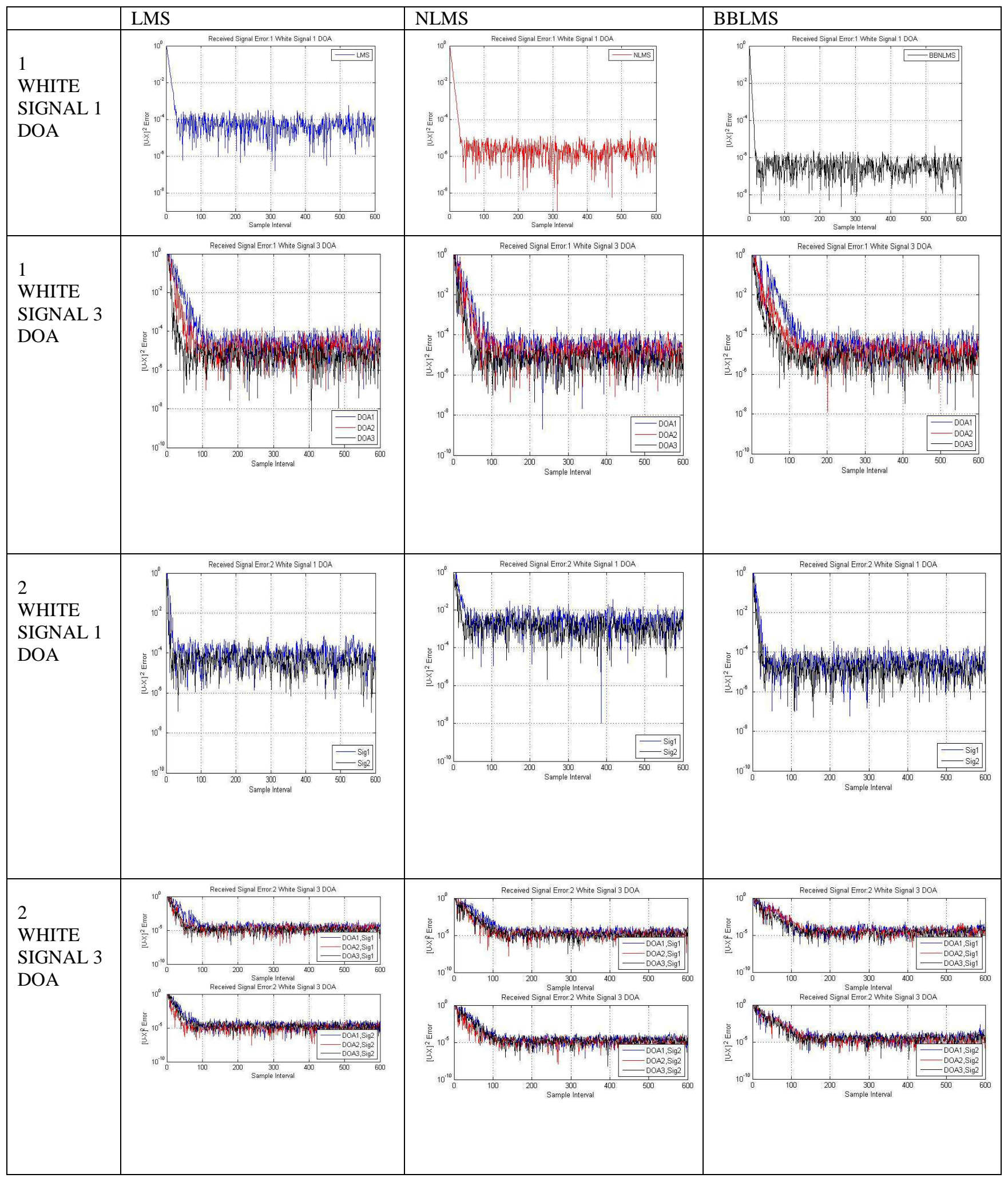

Fig-5: Typical Received signal errors of various signals with different DOA's for LMS,NLMS, BBNLMS algorithms 


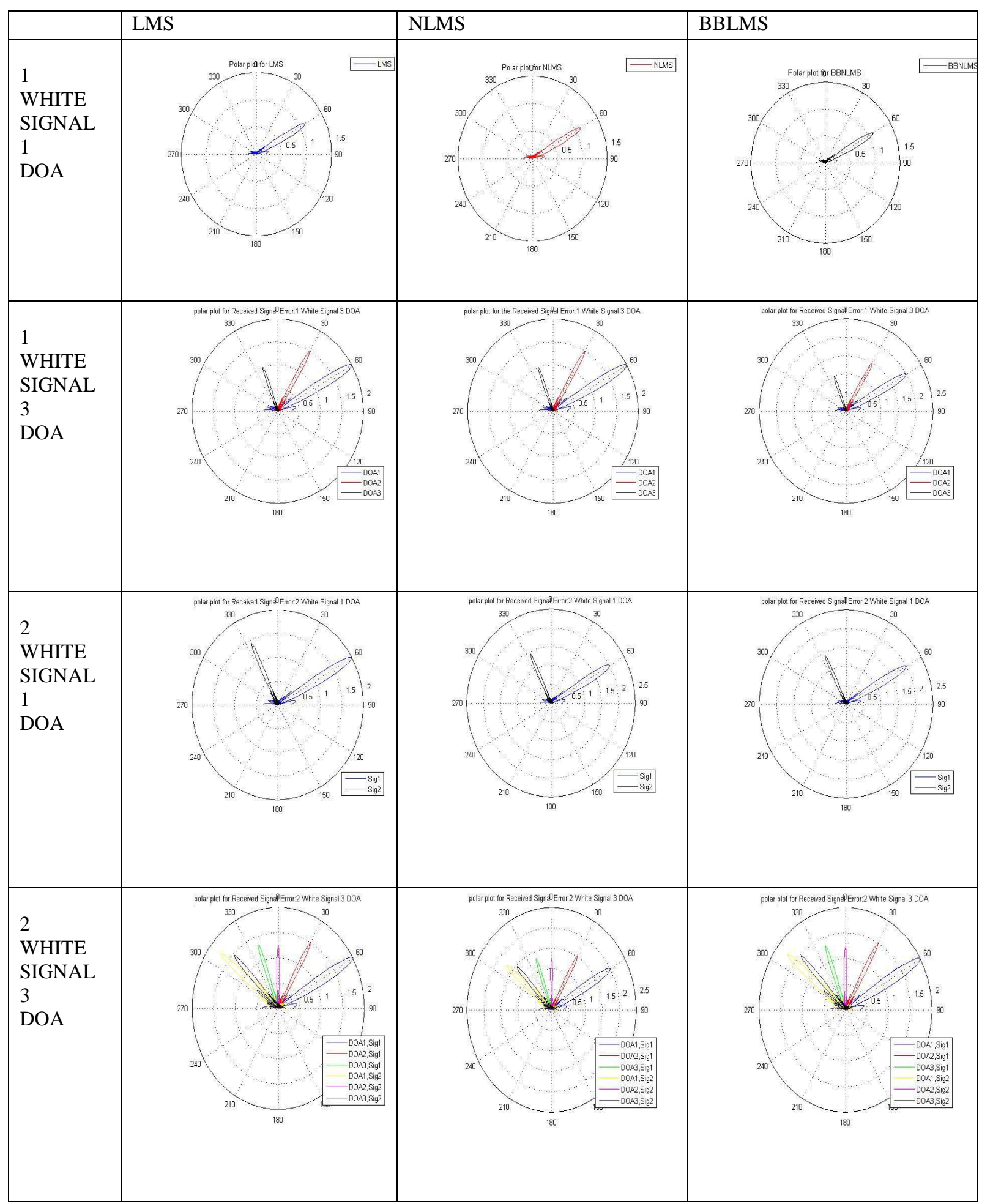

Fig-6: Polar plot of the various signals with different DOA's for LMS, NLMS, BBNLMS algorithms 


\section{CONCLUSIONS}

In this paper several beam forming algorithms are presented with some simulations and results. Using NMLS and BBNLMS algorithms, compared to LMS algorithm, a $40 \%$ and $55 \%$ increase in overall convergence rate is achieved in a multi user multipath environment. With regards to beam patterns, NLMS and BBNLMS algorithms are able to steer beams in the direction of the desired signal and place nulls elsewhere.

\section{REFERENCES}

[1] Carl B. Dietrich, Jr., Warren L. Stutzman, Byung-Ki Kim, and Kai Dietze, "Smart Antennas in Wireless Communications: Base- Station Diversity and Handset Beam forming" lEEE Antennas and Propagation Magazine, Vol. 42, No. 5, October 2000.

[2] Michael Chryssomallis, "Smart Antennas" lEEE Antennas and Propagation Magazine, Vol. 42, No. 3, June 2000.

[3] G.S.N. Raju, Antennas and Wave propagation, Pearson International Publications, Singapore,2010.

[4] R. E. Collin and F. J. Zucker, Antenna Theory, Mc-Graw Hill, New York, 1969.

[5] R. E. Collin, Antennas and Radiowave Propagation, McGraw Hill, New York, 1985.

[6] Frank Gross, "Smart Antenna For Wireless Communication" Mcgraw-hill, September, 2005

[7] Angeliki Alexiou and Martin Haardt, "Smart Antenna Technologies for Future Wireless Systems: Trends and Challenges", IEEE Communication Magazine, Vol. 42, No.9, pp. 90-97, September 2004.

[8] Bernard Widrow, Samuel D. Stearns, "Adaptive Signal Processing", Pearson Education Asia, Second Indian Reprint, 2002.

[9] Symon Haykin, "Adaptive filter theory", Forth edition, Pearson Education, Asia, 2002.

[10] S. D. Blostein and H. Leib, "Multiple antenna systems: Role and impact in future wireless access," IEEE Comm. Mag., vol. 41, no. 7, pp. 94-101, July 2003.

[11] J. H. Winters, "Smart antennas for wireless systems," IEEE Personal Comm., vol. 5, no. 1, pp. 23-27, Feb. 1998.

[12] A. J. Paulraj, D. Gesbert, and C. Papadias, "Smart antennas for mobile communications," Encyclopedia for Electrical Engineering, John Wiley Publishing Co., 2000, pp. $1-15$.

[13]V. Kalinichev, "Analysis of beam-steering and directive characteristics of adaptive antenna arrays for mobile communications," IEEE Antennas Propagat. Mag., vol. 43, no. 3, June 2001.

[14] E. Charpentier and J. Laurin, "An implementation of a directionfinding antenna for mobile communications using a neural network," IEEE Trans. Antennas Propagat., vol. 47, no. 7, July 1999

[15] M. S. Choi, G. Grosskopf, D. Rodhe, B. Kuhlow, G. Przyrembel, and H. Ehlers, "Experiments on DOA-estimation and beam forming for $60 \mathrm{GHz}$ smart antennas," Proc. Vehicular Technology Conference, 2003 (VTC 2003-Spring), 21-24 April 2003, Jeju, Korea, vol. 2, pp. 1041-1045.

\section{BIOGRAPHIES}

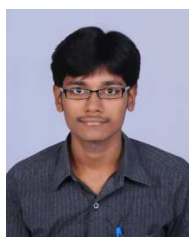

Amarnadh Poluri has obtained B.Tech degree from Prasad Institute of Technology and Sciences affiliated to JNTUK in the year 2011. Now he is pursuing M.Tech Degree in the Department of Electronics \& Communications, Vignan's institute of Information and Technology, Visakhapatnam. He is interested in the fields of wireless and mobile communication, signal processing.

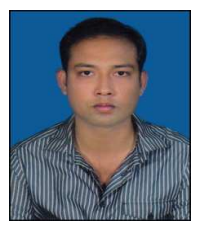

Ashish Kumar has obtained B.Tech degree from Trident academy of technology affiliated to BPUT in the year 2010.he obtained M.Tech degree from Birla Institute of Technology Mesra, Ranchi in the year 2013. Presently he is working as an Asst Professor in the department of Electronics \& Communication Engineering, Vignan's Institute of Information Technology, and Visakhapatnam. He is interested in the fields of wireless communication, signal processing 